\title{
Different roles of matrix metalloproteinase 2 in osteolysis of skeletal dysplasia and bone metastasis (Review)
}

\author{
XIUMAO LI ${ }^{1,2}$, LIBIN JIN ${ }^{1,2}$ and YANBIN TAN ${ }^{1,2}$ \\ ${ }^{1}$ Department of Orthopedics, The Second Affiliated Hospital, Zhejiang University School of Medicine; \\ ${ }^{2}$ Orthopedics Research Institute of Zhejiang University, Hangzhou, Zhejiang 310009, P.R. China
}

Received June 30, 2020; Accepted November 2, 2020

DOI: $10.3892 / \mathrm{mmr} .2020 .11708$

\begin{abstract}
Matrix metalloproteinase 2 (MMP2) is a well-characterized protein that is indispensable for extracellular matrix remodeling and other pathological processes, such as tumor progression and skeletal dysplasia. Excessive activation of MMP2 promotes osteolytic metastasis and bone destruction in late-stage cancers, while its loss-of-function mutations result in the decreased bone mineralization and generalized osteolysis occurring progressively in skeletal developmental disorders, particularly in multicentric osteolysis, nodulosis and arthropathy (MONA). Either upregulation or downregulation of MMP2 activity can result in the same osteolytic effects. Thus, different functions of MMP2 have been recently identified that could explain this observation. While MMP2 can degrade bone matrix, facilitate osteoclastogenesis and amplify various signaling pathways that enhance osteolysis in bone metastasis, its role in maintaining the number of bone cells, supporting osteocytic canalicular network formation
\end{abstract}

Correspondence to: Dr Xiumao Li, Department of Orthopedics, The Second Affiliated Hospital, Zhejiang University School of Medicine, 88 Jiefang Road, Hangzhou, Zhejiang 310009, P.R. China E-mail: simonzju@zju.edu.cn

Abbreviations: BSP, bone sialoprotein; CatK, cathepsin K; ECM, extracellular matrix; ERK, extracellular signal-regulated kinase; LRP, lipoprotein receptor-related protein; MAFB, $\mathrm{V}$-maf musculoaponeurotic fibrosarcoma oncogene ortholog B; M-CSF, macrophage colony-stimulating factor; MMP, matrix metalloproteinase; MMPI, matrix metalloproteinase inhibitor; MONA, multicentric osteolysis, nodulosis and arthropathy; Naa10p, N- $\alpha$-acetyltransferase 10 protein; ObRb, leptin receptor isoform b; OPG, osteoprotegerin; OPN, osteopontin; PDGF-BB, platelet-derived growth factor type BB; PTHrP, parathyroid hormone-related protein; RANKL, receptor activator of $\mathrm{NF}-\kappa \mathrm{B}$ ligand; RBMS3, RNA binding protein 3; TIMP, tissue inhibitors of metalloproteinase; TRAP, tartrate-resistant acid phosphatase; VAO, vessel-associated osteoclast; WS, Winchester syndrome; WT, wild-type

Key words: matrix metalloproteinase 2, osteolysis, skeletal dysplasia, bone metastasis and suppressing leptin-mediated inhibition of bone formation has been implicated in osteolytic disorders caused by MMP2 deficiency. Furthermore, the proangiogenic activity of MMP2 is one of the potential mechanisms that are associated with both pathological situations. In the present article, the latest research on MMP2 in bone homeostasis is reviewed and the mechanisms underlying the role of this protein in skeletal metastasis and developmental osteolysis are discussed.

\section{Contents}

1. Introduction

2. MMP2 in metastatic osteolysis

3. MMP2 in hereditary skeletal dysplasia

4. Mechanisms underlying the role of MMP2 in metastatic osteolysis

5. Mechanisms underlying the role of MMP2 in developmental osteolysis

6. Conclusions

\section{Introduction}

Osteolysis is a common manifestation of bone metastasis and skeletal developmental disorders (1). Bone resorption and formation is a lifelong process that dynamically occurs in the skeletal system and is essential for regulating skeletal growth, repairing bone damage and maintaining mineral homeostasis (2). Either temporal or spatial imbalance of osteoblastic bone formation and osteoclastic bone resorption may lead to progressive osteopenia and subsequently result in osteolysis $(3,4)$. Matrix metalloproteinases (MMPs), including MMP2, have been reported to play key roles in osteolysis by degrading bone extracellular matrix (ECM) and modulating osteoclastic bone resorption (5).

MMP2 is crucial to cancer progression, invasion and metastasis. In many late-stage cancers like breast and prostate cancers, osteolytic bone metastases are frequently observed accompanied with aberrant activation of MMP2 expression $(6,7)$. However, in skeletal developmental disorders, particularly in multicentric osteolysis, nodulosis and arthropathy (MONA), defective or reduced enzyme activity of MMP2 is associated with decreased bone mineralization 
and generalized osteolysis (8). It is interesting to notice that either upregulation or downregulation of MMP2 activity can cause bone loss and osteolysis, suggesting that the mechanism underlying MMP2-related osteolysis is intricate and may differ in different situations. In this article, recent studies on the functions of MMP2 in bone tissues are reviewed and the mechanisms underlying the role of MMP2 in osteolysis that occur in bone metastases and skeletal developmental disorders are summarized. Furthermore, the potential differences between the roles of MMP2 in these two pathological conditions are discussed.

\section{MMP2 in metastatic osteolysis}

Metastasis to the skeletal system is one of the hallmarks of most malignancies (9). It occurs in up to $70 \%$ of patients with cancer, brings great difficulties to disease treatment, and seriously affects patient prognosis $(10,11)$. Due to its ability to cleave and degrade the ECM and basement membrane, the active form MMP2 plays a prominent role in tumor progression and metastasis. MMP2 activity is controlled at multiple levels, from transcriptional regulation, post-translational modification and secretion, to zymogen activation, inhibitor modulation and protein degradation (Fig. 1) (12). The activation of MMP2 predominantly occurs on the cell surface and relies on the coordinated regulation of type-1 membrane MMP (MT1-MMP, also known as MMP14) and tissue inhibitor of metalloproteinase 2 (TIMP-2) (13). Overexpression of MMP2 is usually detected in cancer, and its protein expression levels positively correlate with factors that indicate a poor prognosis, such as poor differentiation, metastasis to secondary organs and chemotherapy resistance $(14,15)$. For instance, in breast cancer, increased expression of MMP2 protein is associated with Twist1-induced invasion and metastasis, whereas decreased MMP2 protein expression levels are involved in RNA binding motif single-stranded interacting protein 3 (RBMS3)-mediated tumor suppression via the RBMS3/Twist1/MMP2 axis (16). Similarly, the protein expression level of MMP2 in pulmonary tumors is notably elevated, which affects the metastatic properties of tumors through a variety of signaling pathways, such as the sphingolipid and ephrin receptor-signaling pathway (17). MMP2 silencing using small interfering RNA, which downregulates the expression of MMP2 both at the protein and mRNA levels, can effectively inhibit the growth and metastasis of non-small cell lung cancer (18). Furthermore, overexpression and hyperactivation of MMP2 can facilitate multiple steps of the metastatic cascade (including intravasation, extravasation, pre-metastatic niche remodeling and regulatory interaction between tumor cells and bone microenvironment) during the invasion of malignant tumors to bone, mostly resulting in osteolytic lesions (19).

Among all manifestations of bone metastasis, osteolysis is one of the most common features of late-stage cancer (20). Extension of osteolytic destruction may lead to pathologic fracture, which occurs in $\sim 10-30 \%$ of all bone metastases (4). Molecules and signaling pathways associated with communication between tumor cells and bone cells serve a role in the pathogenesis of osteolytic metastasis, including parathyroid hormone-related protein (PTHrP), IL-6, IL-11, TNF- $\alpha$, TGF- $\beta$, VEGF and MMPs $(21,22)$. While most members of the MMP family are involved in tumorigenesis and metastasis, MMP2 and MMP9 are thought to be closely associated with bone metastasis and osteolysis. A study on prostate cancer-related bone metastasis demonstrated that integrin $\alpha v \beta 6$ could promote cancer cell-mediated bone destruction by selectively increasing the catalytic activity of MMP2 and the degradation of bone matrix (23). Similarly, thrombospondin-2-induced downregulation of microRNA-376c in prostate cancer cells increases MMP2 expression and bone metastasis (24). In breast cancer, MMP2 upregulates ERK signaling and reverses the receptor activator of $\mathrm{NF}-\kappa \mathrm{B}$ ligand (RANKL)/osteoprotegerin (OPG) ratio, thus promoting bone resorption and metastasis (5).

The role of MMP2 in osteolysis during tumor metastasis has been further confirmed by the application of matrix metalloproteinase inhibitors (MMPIs) in tumor therapy (25). Insufficient control of MMP2 activity by TIMPs (especially TIMP-2, -3 and -4), the endogenous inhibitors of MMPs, would result in dysregulation of tissue remodeling and tumors (26). When synthesized MMPIs are utilized, they are expected to disrupt the 'vicious cycle' that occurs in the spread of tumor cells to bone. To date, four generations of MMP2-targeting inhibitors have been identified or designed for cancer therapy, namely the first generation of hydroxamate-based inhibitors, the second generation of non-hydroxamate-based inhibitors, the third generation of catalytic domain (non-zinc binding) inhibitors, and the fourth generation of allosteric and exosite inhibitors $(26,27)$. Although clinical trials involving MMPIs have not achieved the expected results in cancer therapy, preclinical results from tumor experiments using MMP2 inhibitors, such as FYK-1388, chlorotoxin and the monoclonal antibody 9E8, support the osteolytic effect of MMP2 in bone metastases (28). For example, 2-[[(4-phenoxyphenyl) sulfonyl] methyl]-thiirane (SB-3CT), a selective inhibitor of gelatinase that inhibits MMP2 (also known as gelatinase A) strongly and MMP9 (gelatinase B) weakly, has been shown to inhibit intra-bone growth of prostate cancer cells and bone degradation $(29,30)$.

\section{MMP2 in hereditary skeletal dysplasia}

Intriguingly, while numerous studies in cancer have demonstrated that higher MMP2 activity correlates with more severe osteolytic destruction in metastatic lesions, MMP2 hypoactivity also leads to bone loss and osteolysis during skeletal growth and development (31). The most well-known evidence for MMP2 deficiency-related osteolysis comes from inherited developmental diseases, particularly MONA and Winchester syndrome (WS), which are caused by inactive mutations in the MMP2 and MMP14 genes, respectively $(8,32)$. Both of them are autosomal recessive disorders characterized by highly similar phenotypes of progressive multicentric osteolysis and arthritis. Although not directly associated with MMP2 mutation, the pathogenesis of WS is thought to be linked with deficient MMP2 activity, since intact function of MMP14 is critical for activation of MMP2 (33).

As an extremely rare genetic disease with only 46 cases and 23 mutations from 28 families reported in the literature, no exact epidemiological statistics of MONA, have been described (34). Typical clinical manifestations of MONA 


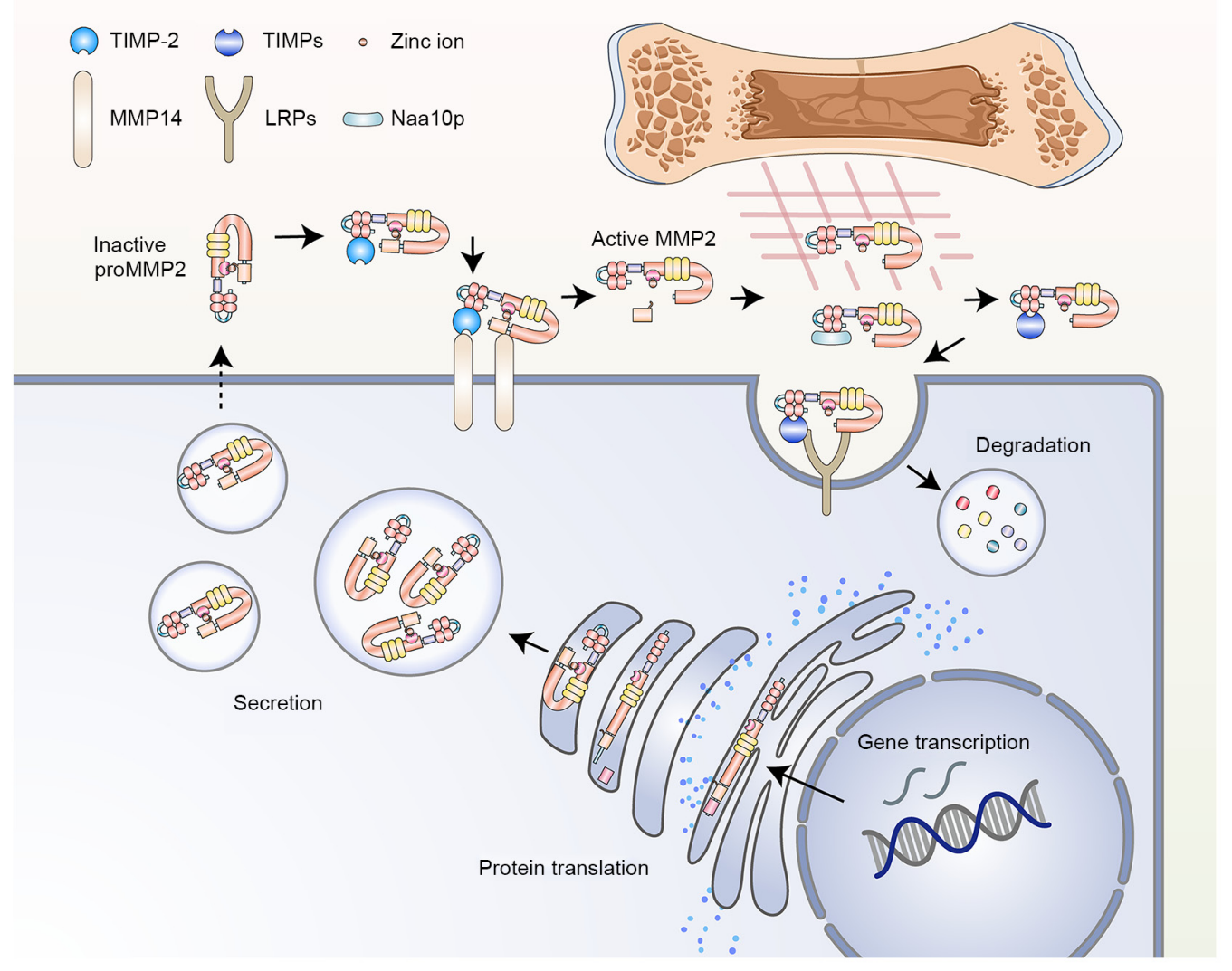

Figure 1. Expression and activation of MMP2. After being secreted, proMMP2 is activated by the collaboration between TIMP-2 and MMP14. Extracellular proMMP2 binding with the C-terminal tail of TIMP-2 is indirectly linked to the cell membrane-anchored MMP14 through the interaction between the $\mathrm{N}$-terminal domain of TIMP-2 and the catalytic domain of MMP14. This proMMP2/TIMP-2/MMP14 complex is recruited to another adjacent MMP14 that converts proMMP2 to the active enzyme form. On the other hand, MMP2 activity can also be inhibited by TIMP-2 when its active site binds to the N-terminal inhibitory domain of TIMP-2. While several TIMPs inhibit the activity of MMP2 and lead to LRP-mediated endocytosis and degradation, Naa10p can stabilize MMP2 protein. LRP, lipoprotein receptor-related proteins; MMP, matrix metalloproteinase; Naa10p, N- $\alpha$-acetyltransferase 10 protein; TIMP, tissue inhibitors of metalloproteinase.

consist of extensive osteopenia and osteolysis (most prominent in carpal and tarsal bones), progressive osteoarthropathy, and subcutaneous nodules on the palms and soles (35). In general, most children with MMP2 mutations are apparently healthy at birth, but signs of skeletal deformities begin from six months to eleven years old. Thereafter, features of arthropathy gradually appear, including joint pain, joint contracture and swelling of distal extremities, and other symptoms then progressively develop, such as coarse facies and gum hypertrophy (36). Vanatka et al (37) reported a case of MONA with long-term follow-up, in which the pathological changes of the bones over a 23-year period were striking and the pattern of bone loss was progressive and periarticular. Furthermore, $\sim 75 \%$ of mutations from the reported MONA cases occur in the catalytic domain or have detrimental effects on its catalytic function, resulting in impaired MMP2 activity $(34,38)$. Consequently, although individual differences in clinical manifestations exist among MONA patients, almost all of them contain signs of bone loss and osteolysis, indicating that loss of MMP2 accounts for impaired bone integrity and mineral homeostasis during skeletal growth and development.

To date, there is a conflict between the results obtained in tumor research and developmental research regarding the relationship between MMP2 and osteolysis. Based on the current understanding of the structure and function of
MMP2 protein, the finding that MMP2 deficiency causes bone loss and osteolytic phenotype seems counterintuitive. It was hypothesized that a reduction in MMP2 activity may lead to bone overgrowth rather than bone loss (39). However, the role of MMP2 in osteolysis may be more complex than previously thought, and studies have been conducted to investigate potential explanations for how MMP2 functions in opposite ways in these two pathological conditions.

\section{Mechanisms underlying the role of MMP2 in metastatic osteolysis}

MMP2 promotes tumor-induced osteolysis involving spatial accessibility achieved through cleavage of collagen on the bone surface and stimulation of osteoclastic bone resorption (40). In bone tissue, MMP2 is mainly expressed by osteoblasts under the regulation of various factors, such as FasL (41). During bone metastasis, tumor cells and tumor stromal cells become another important source of MMP2, and thus markedly promote osteoclastogenesis and osteoclastic bone resorption (Fig. 2). Expression of MMP2 has been confirmed in both human breast cancer cell lines and primary breast tumors, and high protein levels of MMP2 are associated with increased risk of bone metastasis (5). After being released into the extracellular compartment, although MMP2 may not directly 


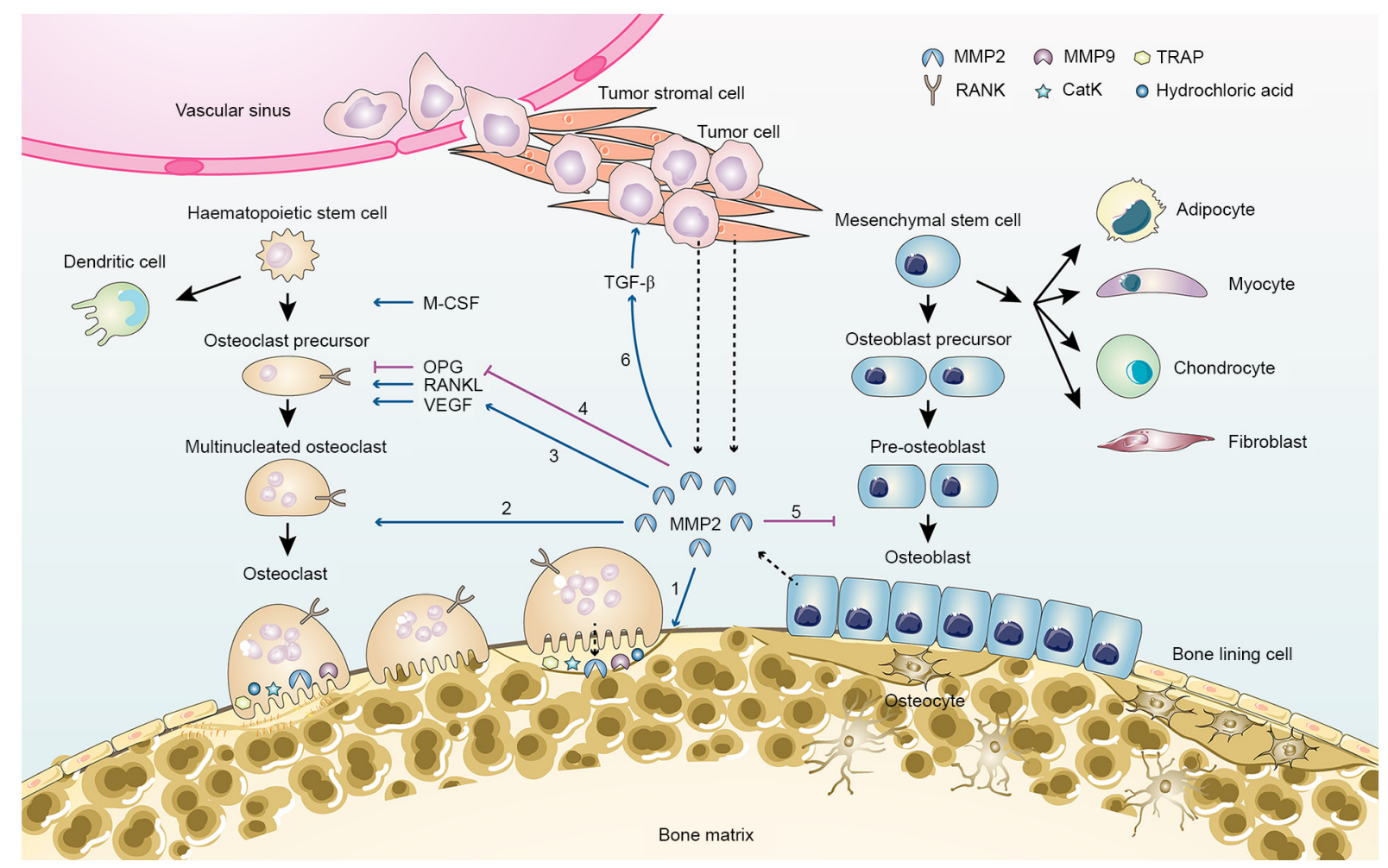

Figure 2. MMP2 in bone remodelling and tumor metastasis-related bone loss. MMP2 is primarily secreted by osteocytes, osteoblasts, tumor stromal cells and tumor cells (dashed arrow). MMP2 contributes to bone loss via several pathways: 1, Degradation of collagen and osteoid seam on the bone surface; 2, recruitment of pre-osteoclasts and differentiation of osteoclasts; 3, increased VEGF activation, which promotes osteoclastogenesis; 4 , inhibition of OPG expression and increase in the RANKL/OPG ratio; 5 , inhibition of osteoblast differentiation; 6 , activation of growth factors, such as TGF- $\beta$. CatK, cathepsin K; M-CSF, macrophage-colony-stimulating factor; MMP, matrix metalloproteinase; Naa10p, $\mathrm{N}$ - $\alpha$-acetyltransferase 10 protein; OPG, osteoprotegerin; RANKL, receptor activator of NF-kB ligand; TRAP, tartrate-resistant acid phosphatase.

participate in degradation of mineralized bone matrix, it can cleave the collagen and osteoid seam that covers the bone surface, activate osteolytic factors, and facilitate osteoclasts recruitment and attachment $(4,41)$. Activated MMP2 can suppress the expression of OPG in osteoblasts and increase the RANKL/OPG ratio, thereby contributing to osteoclast differentiation (4). In addition to its role in promoting osteoclastic resorption, MMP2 can inhibit the later phases of osteoblast differentiation by shedding the immune costimulatory molecule B7-H3 from the cell membrane of osteoblasts (42). Moreover, mature osteoclasts may also synthesize MMP2 and release it along with MMP9 and protons into the local extracellular compartment to digest bone matrix $(43,44)$.

MMP2 also provides signal amplification for the "vicious cycle' within the tumor-bone microenvironment by regulating the activity and bioavailability of various growth factors such as TGF- $\beta$, PTHrP and RANKL (19). In particular, the communication between MMP 2 and TGF- $\beta$ is of particular importance for this signaling crosstalk between tumor cells and bone cells. Expression of TGF- $\beta$ has been confirmed in a large panel of cancer types with metastatic properties (45). Furthermore, a large amount of TGF- $\beta$ could be released from the bone matrix degraded by MMPs during bone resorption, including MMP2 and MMP9, and this increase in TGF- $\beta$ from osteolytic lesions after metastatic destruction has been observed in many mouse models of osteolytic bone metastasis (45). Excessive TGF- $\beta$ signaling in turn stimulates the release of PTHrP and IL-11 from tumor cells and promotes osteolytic bone destruction $(4,46)$.
TGF- $\beta$ can regulate bone remodeling and maintenance of bone mass via its dual effects on osteoblasts (47). It potentiates the recruitment of mesenchymal stem cells and promotes the early differentiation of osteoblast precursors by inducing Runx2 expression, which is required for the initiation of osteoblast differentiation and inhibition of late differentiation of osteoblasts (47). In addition to its effect on RANKL stimulation and OPG expression, hyperactivation of TGF- $\beta$ signaling has been reported to directly enhance the bone-resorptive activity of osteoclasts (48). Moreover, increased TGF- $\beta$ levels can promote late-stage cancer by activating surrounding cancer-associated fibroblasts and stellate cells, stimulating angiogenesis and inducing immunosuppression, thus facilitating the proliferation and invasion of cancer cells in osteolytic lesions (49).

The proangiogenic effect of MMP2 is another critical mechanism for osteolysis during bone metastasis. Many members of the MMP family have been implicated in tumor neovascularization and lymphangiogenesis. For instance, MMP1 induces the expression of VEGFR2, while MMP7 acts as a regulator for the VEGF pathway and the degradation of soluble VEGFR1 (50). Specifically, MMP2 and MMP9 cooperate tightly in neoplastic angiogenesis by modulating the dynamic remodeling of ECM. Studies have revealed that elevated MMP2 expression is correlated with increased activation of TGF- $\beta$ and VEGF in the metastatic lesions $(22,51)$. The bioactive VEGF, basic fibroblast growth factor (bFGF) and TGF- $\beta$ can not only induce angiogenesis by signaling through their respective receptors on endothelial cells, but also stimulate 


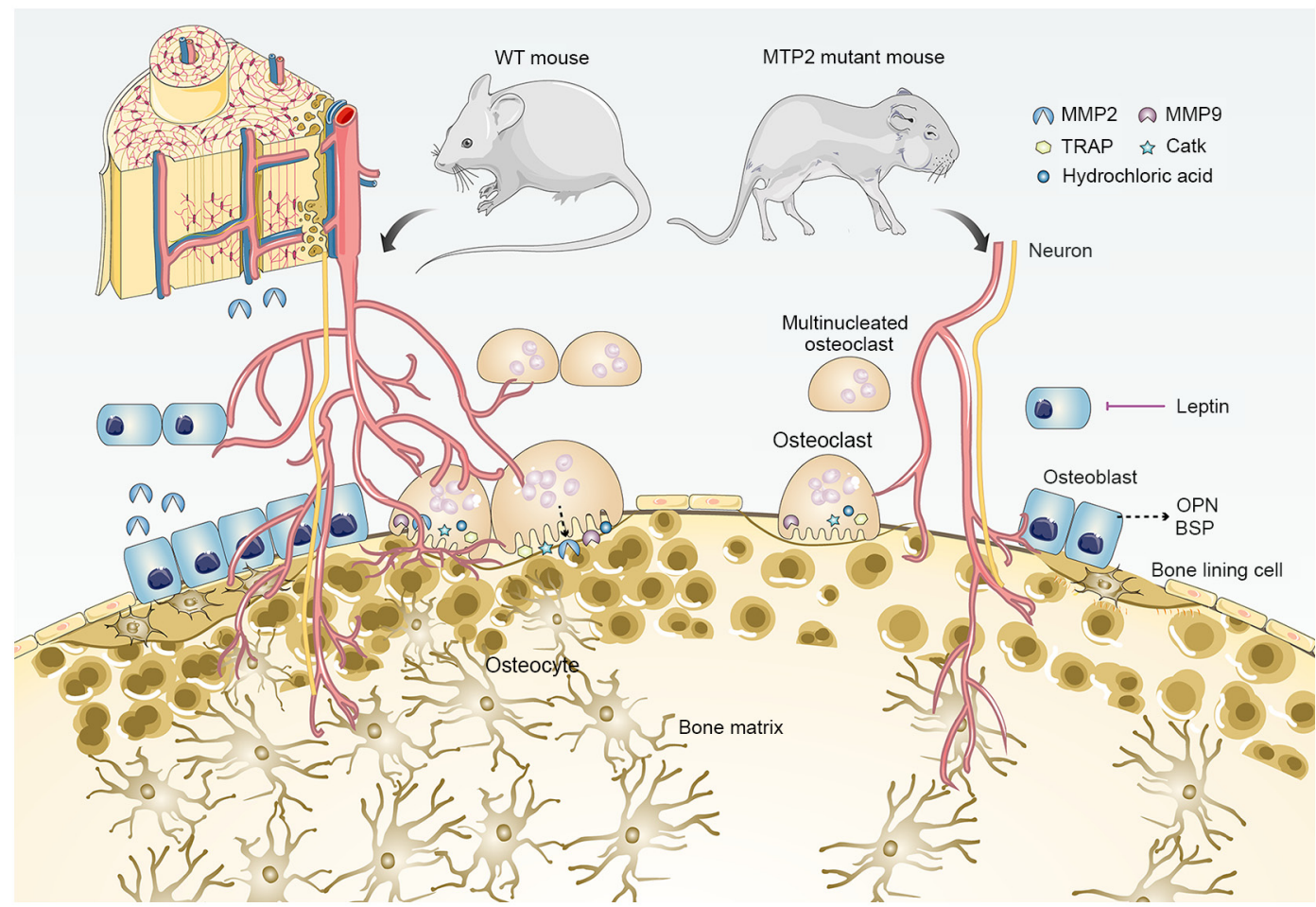

Figure 3. MMP2 deficiency and bone loss. Based on studies of MMP2 mutant mouse models, several phenotypes related to bone loss have been reported, including: 1, Reduced growth rate during development (although the overall anatomical structure of mutant mice is normal in adulthood); 2, progressive loss of bone mineral density and articular cartilage destruction; 3 , decreased connectivity density of trabeculae; 4 , age-dependent reduction in the number of osteoblasts and osteoclasts; 5, suppressed formation of osteocytic canalicular network; 6, upregulated transcription of OPN and BSP; 7, enhanced leptin-mediated inhibition of bone formation. BSP, bone sialoprotein; CatK, cathepsin K; MMP, matrix metalloproteinase; OPG, osteoprotegerin; OPN, osteopontin; TRAP, tartrate-resistant acid phosphatase; WT, wild-type.

the secretion of MMP2 and MMP9-containing vesicles from endothelial cells, thereby contributing to the upregulation of proteolytic activity in the metastatic microenvironment (50). Besides, in vivo studies have confirmed that angiogenesis and tumor progression were inhibited in mice lacking MMP2, according to a dorsal air sac assay using melanoma cells and lung carcinoma cells (52).

\section{Mechanisms underlying the role of MMP2 in developmental osteolysis}

How does MMP2 deficiency contribute to developmental osteolysis? As aforementioned, high activity of MMP2 is associated with increased bone resorption, suggesting that MMP2 mainly functions as a negative modulator for bone remodeling. In addition, there are also some studies supporting that MMP2 positively associates with bone formation. For instance, Saran et al (53) demonstrated that MMP2 expression was upregulated in newly formed bone tissue, whereas MMP9 expression was reduced according to their reamed tibia medullary canal mouse model. They proposed that MMP2 facilitated bone remodeling and maturation probably by removing organic bone content. Since the role of MMP2 in bone remodeling is controversial, there may be other mechanisms that are responsible for the pathogenesis of developmental osteolysis resulted from MMP2 deficiency.

Insight from MMP2-deficient mice. In order to understand how MMP2 affects skeletal development, researchers have developed several mutant mouse models (12). MMP2 knockout mice were first generated by Itoh et al (54) via targeted replacement of the promoter and the first exon of $M m p 2$ gene. Although the mutant mice develop normally without any gross anatomical abnormalities, they have a significantly slower growth rate and exhibit several attenuated features of human MONA, such as progressive loss of bone mineral density and articular cartilage destruction (Fig. 3) (55). Moreover, opposing bone phenotypes of decreased mineral density in long bones but increased volume in cranium have also been described (56). In addition, MMP2 deficiency in mice reduces the connectivity density of trabeculae, and impairs bone remodeling but not cartilage remodeling during fracture repair $(57,58)$. Based on the aforementioned observations, several novel functions of MMP2 in skeletal development have been proposed, such as maintenance of the number of osteoblasts and osteoclasts in an age-dependent manner, supporting the formation of osteocytic canalicular network, and controlling the transcription of osteopontin (OPN) and bone sialoprotein (BSP) $(39,55,56)$. However, evidence from a detailed mechanism that comprehensively interprets the consequences of MMP2 deficiency is still insufficient.

Comparative studies of similar diseases have provided another conclusion regarding the function of MMP2 in developmental osteolysis. In addition to MONA and WS, there is a third type of osteolytic skeletal dysplasia called multicentric carpal-tarsal osteolysis syndrome, which is caused by mutations in v-maf musculoaponeurotic fibrosarcoma oncogene ortholog B (MAFB) (59). By investigating the characteristics 


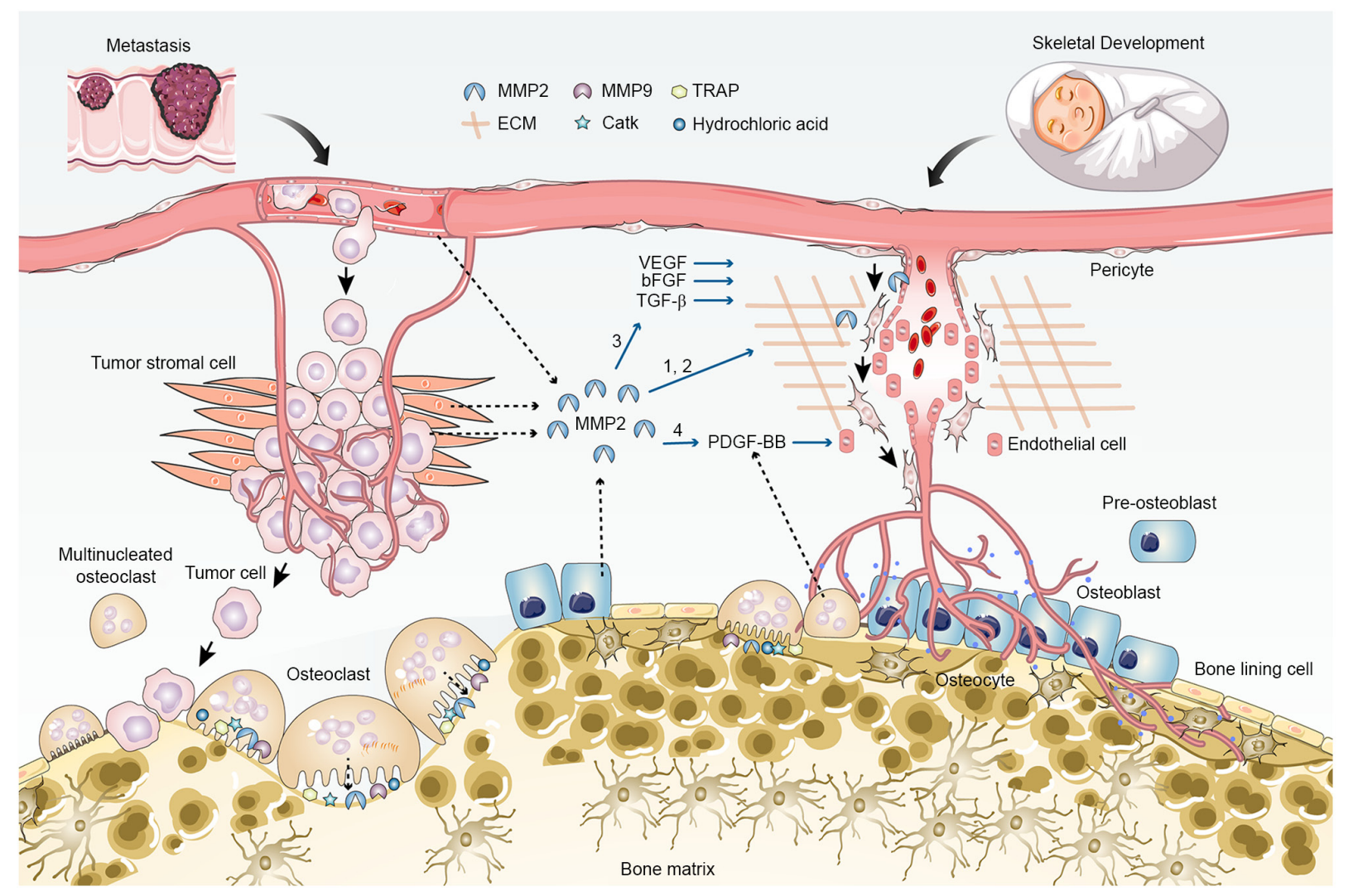

Figure 4. Proangiogenic role of MMP2 in tumor metastasis and skeletal development. MMP2 promotes angiogenesis in multiple ways: 1, Degradation of vascular basement membrane and extracellular matrix, and cell-cell adhesion cleavage; 2, Detachment of pericytes and migration of endothelial cells; 3 , increased release and activation of ECM-bound angiogenic growth factors, such as VEGF, bFGF and TGF- $\beta$; 4 , potential increase in the secretion of PDGF-BB through induction of osteoclast differentiation and maturation. Angiogenesis is coupled with osteogenesis during skeletal development, but supports tumor cell-induced osteolysis during metastasis. bFGF, basic fibroblast growth factor; CatK, cathepsin K; ECM, extracellular matrix; MMP, matrix metalloproteinase; TRAP, tartrate-resistant acid phosphatase.

of those so-called human carpal-tarsal osteolysis disorders, Lazarus et al (60) suggested that osteoclast-mediated resorption was not sufficient to explain the distribution pattern of developmental osteolysis. Indeed, MMP2, MMP14 and MAFB were expressed in chondrocytes from the earliest stages of subarticular endochondral ossification. Based on the hypothesis that the endochondral ossification in subarticular regions is different from that in archetypical physeal regions, the authors suggested that abnormal peri-articular skeletal development and modeling, rather than the excessive osteoclastic bone resorption, was responsible for site-specific osteolysis (60).

Proangiogenic function of MMP2. In addition to its direct effect on bone cells, the role of MMP2 in angiogenesis may be a key mechanism explaining its different functions in tumor metastasis and bone dysplasia (Fig. 4). MMP2 can intervene in angiogenesis in multiple ways. Firstly, MMP2 enables the detachment of pericytes and migration of endothelial cells by degrading the vascular basement membrane and ECM and cleaving endothelial cell-cell adhesions (61). Secondly, the proteolytic activity of MMP2 can promote the release and activation of ECM-bound angiogenic growth factors, including VEGF, bFGF and TGF- $\beta(50,62)$. The proangiogenic function of MMP2 has been confirmed in MMP2-deficient mice $(63,64)$. According to a previous study using a hindlimb ischemia model, both collateral vessel development and capillary formation were suppressed in MMP2-deficient mice, and the invasive and proliferative abilities of endothelial cells cultured from mutant mice were significantly impaired (63). Angiogenesis is a basic condition necessary to support both tumor progression and bone formation. Therefore, in spite of the same proangiogenic effect, upregulation of MMP2 indirectly leads to osteolytic destruction by promoting tumor cell proliferation and invasion during bone metastasis, while its downregulation directly impairs bone formation during osteogenesis, due to reduced blood supply.

Recent studies have provided some new insights into the relationship between MMP2 and angiogenesis. The endothelial cells of type-H vessels are intimately associated with both bone modeling and remodeling owing to their ability to support bone tissues with indispensable nutrients and can secrete MMPs that modulate ECM and vessel growth (65). MMP9 and MMP2 release participates in the regulation of cartilage resorption and bone vasculature, respectively. In particular, the promoting role of MMP2 in osteoclast differentiation is likely to facilitate the secretion of platelet-derived growth factor type BB (PDGF-BB), thereby stimulating the formation of type-H vessels (66). Moreover, non-resorbing vessel-associated osteoclasts (VAOs), which are critical for anastomoses of type-H vessels and blood vessel-directed elongation of bones, are also regulated by RANKL signaling in 
type-H endothelial cells expressing high levels of MMP2 (67). It is possible that deficiency in MMP2 also leads to disrupted orientation of angiogenic blood vessels and contorted bone shape by affecting type-H endothelial cells and VAOs.

Other potential mechanisms. Additional factors may be involved in modulating the function of MMP2 in skeletal development, especially through the central nervous system. There is increasing evidence for cross-talk between the brain and bone through several pathways, including hormonal signals and cell-cell communication between neurons and bone cells (3). For instance, leptin is an anorexigenic hormone that is predominantly secreted from white adipose tissue and is essential for metabolic regulation. It can activate the sympathetic nervous system through leptin receptor isoform $b$, also known as obesity receptor isoform b (ObRb), on hypothalamic neurons to inhibit bone formation, while its direct interaction with $\mathrm{ObRb}$-positive bone cells may increase their growth $(68,69)$. A study on obesity has recently identified that activated MMP2 in hypothalamus modulates leptin-mediated signaling by cleaving the extracellular domain of ObRb leptin receptors (70). Knockdown of MMP2 restores the expression levels of the leptin receptor and increases the concentration of leptin in hypothalamic neurons (70). Therefore, it may be hypothesized that loss of MMP2 may promote leptin signaling and enhance the leptin-mediated inhibition of bone formation. Further studies are required to verify whether leptin signaling or any other factors of the autonomic system also contribute to skeletal dysplasia and osteolysis caused by MMP2 deficiency.

In addition to excessive osteoclastic bone resorption, dysfunction of osteoblastic bone formation can also give rise to osteopenia and osteolysis during bone development. Metabolic programming is one of the essential factors that control the bone-forming function of osteoblasts, and metabolic diseases such as diabetes mellitus and anorexia nervosa can result in osteoblast dysfunction and bone loss (71). It has been reported that MMP2 deficiency is associated with metabolic disorders, making the affected patients prone to osteolysis and growth retardation (72). In addition to its extracellular proteolytic functions, increasing evidence indicates that MMP2 has considerable intracellular functions, including degradation of intracellular proteins like calcitonin gene-related peptide and nuclear matrix proteins like poly (ADP-ribose) polymerase $(73,74)$. Consequently, loss-of-function mutations in MMP2 may disrupt the energy metabolism of osteoblasts, thereby impairing their bone formation function.

\section{Conclusions}

The molecular and cellular mechanisms involved in MMP2-related osteolysis during tumor metastasis and skeletal development are not fully understood yet. In this review, the most important findings in the field were summarized and classified into three categories based on their relationships with bone formation.

The first set of functions answers to a certain extent how overexpression of MMP2 is associated with osteolysis in bone metastasis (Fig. 2). Some of the relevant functions include: i) Degrading the collagen and osteoid seam on the bone surface; ii) facilitating osteoclast recruitment and osteoclastogenesis; iii) increasing the RANKL/OPG ratio; iv) inhibiting osteoblast differentiation; v) activating growth factors and osteolytic factors, such as TGF- $\beta$ and IL-6; and vi) amplifying the mutual regulatory interactions that occur between tumor cells and the microenvironment. Together, these functions illustrate the role of MMP2 in promoting osteoclastic bone resorption, which provides the framework for understanding the effect of MMP2 on metastatic osteolysis.

The second set of functions is in accord with the phenomena observed in osteolytic disorders caused by MMP2 deficiency (Fig. 3). The application of genetically engineered mouse models of MMP2 deficiency has provided insight into the molecular mechanisms that enable MMP2 to maintain normal bone mass and prevent developmental abnormalities. The prominent findings for this aspect of MMP2 functions include: i) Maintaining the number of bone cells; ii) supporting the formation of the osteocytic canalicular network; iii) controlling the transcription of mineralization-related factors such as OPN and BSP; iv) fostering subarticular endochondral ossification and periarticular bone modeling; and v) suppressing leptin-mediated inhibition of bone formation. However, the question of whether MMP2 is required for energy metabolism in osteoblasts remains to be demonstrated.

The last set of functions takes into account the distinct roles of MMP2 in two pathological conditions. Since both tumors and bones are highly vascularized tissues and angiogenesis is a prerequisite for their development, MMP2 can influence bone metastasis and osteogenesis through its proangiogenic activity (Fig. 4). As aforementioned, in terms of molecular mechanisms for neoplastic angiogenesis, MMP2 facilitates the remodeling of ECM, stimulates the migration of pericytes and endothelial cells, and activates angiogenic growth factors. During bone metastasis, the pre-metastatic niche developed by the neovascular network facilitates metastatic colonization of cancer cells and subsequent bone destruction. These functions of MMP2 also appear to be of particular importance to vasculogenesis during skeletal development; formation of neovascular networks exerts a positive effect on the skeleton. Moreover, several novel concepts that relate MMP2 with angiogenesis and osteogenesis have been highlighted, including type- $\mathrm{H}$ vessels that are tightly coupled with periosteal bone formation, as well as VAOs, which are associated with blood vessel-directed elongation of bones. Nevertheless, the detailed mechanism underlying how MMP2 contributes to osteolysis and how MMP2 performs distinct roles under physiological and pathological conditions remains unclear. In summary, although MMP2 has been shown to be a critical mediator of bone destruction and resorption, it may also serve additional functions that remain to be identified. Furthermore, the mechanistic interactions between MMP2 and other proteases, which may fine-tune and ultimately determine the end-activity of MMP2, require further study. A better understanding of the mechanisms that underlie the functions of MMP2 during osteolytic metastasis and developmental osteolysis would facilitate the exploitation of therapeutics for restoring the phenotypes caused by MMP2 hyperactivity or deficiency.

\section{Acknowledgements}

Not applicable. 


\section{Funding}

This study was funded by a grant from the National Natural Science Foundation of China (grant no. 81702662).

\section{Availability of data and materials}

Data sharing is not applicable to this article, as no data sets were generated or analyzed during the current study.

\section{Authors' contributions}

XL, LJ and YT conceived and designed the study. XL and LJ researched and performed analysis of the literature. XL drafted the manuscript, and YT critically revised important intellectual content of the article. All authors read and approved the final manuscript.

\section{Ethics approval and consent to participate}

Not applicable.

\section{Patient consent for publication}

Not applicable.

\section{Competing interests}

The authors declare that they have no competing interests.

\section{References}

1. Zaidi M: Skeletal remodeling in health and disease. Nat Med 13: 791-801, 2007.

2. Hassan B, Baroukh B, Llorens A, Lesieur J, Ribbes S, Chaussain C, Saffar JL and Gosset M: NAMPT expression in osteoblasts controls osteoclast recruitment in alveolar bone remodeling. J Cell Physiol 233: 7402-7414, 2018.

3. Sato S, Hanada R, Kimura A, Abe T, Matsumoto T, Iwasaki M, Inose H, Ida T, Mieda M, Takeuchi Y, et al: Central control of bone remodeling by neuromedin U. Nat Med 13: 1234-1240, 2007.

4. Battafarano G, Rossi M, Marampon F and Del Fattore A: Cellular and molecular mediators of bone metastatic lesions. Int J Mol Sci 19: 1709, 2018.

5. Ni X, Xia T, Zhao Y, Zhou W, Wu N, Liu X, Ding Q, Zha X, Sha J and Wang S: Downregulation of miR-106b induced breast cancer cell invasion and motility in association with overexpression of matrix metalloproteinase 2. Cancer Sci 105: 18-25, 2014.

6. Ell B, Mercatali L, Ibrahim T, Campbell N, Schwarzenbach H, Pantel K, Amadori D and Kang Y: Tumor-induced osteoclast miRNA changes as regulators and biomarkers of osteolytic bone metastasis. Cancer Cell 24: 542-56, 2013.

7. Huang S, Shao K, Liu Y, Kuang Y, Li J, An S, Guo Y, Ma H and Jiang C: Tumor-targeting and microenvironment-responsive smart nanoparticles for combination therapy of antiangiogenesis and apoptosis. ACS Nano 7: 2860-2871, 2013.

8. Bhavani GS, Shah H, Shukla A, Gupta N, Gowrishankar K Rao AP, Kabra M, Agarwal M, Ranganath P, Ekbote AV, et al: Clinical and mutation profile of multicentric osteolysis nodulosis and arthropathy. Am J Med Genet A 170A: 410-417, 2016.

9. Ragel BT, Mendez GA, Reddington J, Ferachi D, Kubicky CD, Philipp TC, Zusman NL, Klimo P, Hart R, Yoo J and Ching AC: Life Expectancy and metastatic spine scoring systems: An academic institutional experience. Clin Spine Surg 30: 335-342, 2017.

10. Croucher PI, McDonald MM and Martin TJ: Bone metastasis: The importance of the neighbourhood. Nat Rev Cancer 16: 373-386, 2016.
11. Vanek P, Bradac O, Trebicky F, Saur K, de Lacy P and Benes V: Influence of the preoperative neurological status on survival after the surgical treatment of symptomatic spinal metastases with spinal cord compression. Spine (Phila Pa 1976) 40: 1824-1830, 2015.

12. Page-McCaw A, Ewald AJ and Werb Z: Matrix metalloproteinases and the regulation of tissue remodelling. Nat Rev Mol Cell Biol 8: 221-233, 2007.

13. Itoh Y: Membrane-type matrix metalloproteinases: Their functions and regulations. Matrix Biol 44-46: 207-223, 2015.

14. Deryugina EI and Quigley JP: Matrix metalloproteinases and tumor metastasis. Cancer Metastasis Rev 25: 9-34, 2006.

15. Henriet $P$ and Emonard H: Matrix metalloproteinase-2: Not (just) a 'hero' of the past. Biochimie 166: 223-232, 2019.

16. Zhu L, Xi PW, Li XX, Sun X, Zhou WB, Xia TS, Shi L, Hu Y, Ding $Q$ and Wei JF: The RNA binding protein RBMS3 inhibits the metastasis of breast cancer by regulating Twistl expression. J Exp Clin Cancer Res 38: 105, 2019.

17. Merchant N, Nagaraju GP, Rajitha B, Lammata S, Jella KK, Buchwald ZS, Lakka SS and Ali AN: Matrix metalloproteinases: Their functional role in lung cancer. Carcinogenesis 38: 766-780, 2017.

18. Wang H, Guan X, Tu Y, Zheng S, Long J, Li S, Qi C, Xie X, Zhang $\mathrm{H}$ and Zhang Y: MicroRNA-29b attenuates non-small cell lung cancer metastasis by targeting matrix metalloproteinase 2 and PTEN. J Exp Clin Cancer Res 34: 59, 2015.

19. Tauro M and Lynch CC: Cutting to the Chase: How matrix metalloproteinase-2 activity controls breast-cancer-to-bone metastasis. Cancers (Basel) 10: 185, 2018.

20. Coleman RE, Croucher PI, Padhani AR, Clezardin P, Chow E, Fallon M, Guise T, Colangeli S, Capanna R and Costa L: Bone metastases. Nat Rev Dis Primers 6: 83, 2020.

21. Kenkre JS and Bassett J: The bone remodelling cycle. Ann Clin Biochem 55: 308-327, 2018.

22. Mathis KM, Sturgeon KM, Winkels RM, Wiskemann J, De Souza MJ and Schmitz KH: Bone resorption and bone metastasis risk. Med Hypotheses 118: 36-41, 2018.

23. Dutta A, Li J, Lu H, Akech J, Pratap J, Wang T, Zerlanko BJ, FitzGerald TJ, Jiang Z, Birbe R, et al: Integrin $\alpha v \beta 6$ promotes an osteolytic program in cancer cells by upregulating MMP2. Cancer Res 74: 1598-1608, 2014.

24. Chen PC, Tang CH, Lin LW, Tsai CH, Chu CY, Lin TH and Huang YL: Thrombospondin-2 promotes prostate cancer bone metastasis by the up-regulation of matrix metalloproteinase-2 through down-regulating miR-376c expression. J Hematol Oncol 10: 33, 2017.

25. Zhong Y, Lu YT, Sun Y, Shi ZH, Li NG, Tang YP and Duan JA: Recent opportunities in matrix metalloproteinase inhibitor drug design for cancer. Expert Opin Drug Discov 13: 75-87, 2018.

26. Li K, Tay FR and Yiu CKY: The past, present and future perspectives of matrix metalloproteinase inhibitors. Pharmacol Ther 207: 107465, 2020.

27. Fields GB: Mechanisms of action of novel drugs targeting angiogenesis-promoting matrix metalloproteinases. Front Immunol 10: 1278, 2019.

28. Winer A, Adams S and Mignatti P: Matrix metalloproteinase inhibitors in cancer therapy: Turning past failures into future successes. Mol Cancer Ther 17: 1147-1155, 2018.

29. Bonfil RD, Sabbota A, Nabha S, Bernardo MM, Dong Z, Meng H, Yamamoto H, Chinni SR, Lim IT, Chang M, et al: Inhibition of human prostate cancer growth, osteolysis and angiogenesis in a bone metastasis model by a novel mechanism-based selective gelatinase inhibitor. Int J Cancer 118: 2721-276, 2006.

30. Tao P, Fisher JF, Mobashery S and Schlegel HB: DFT studies of the ring-opening mechanism of SB-3CT, a potent inhibitor of matrix metalloproteinase 2. Org Lett 11: 2559-2562, 2009.

31. Martignetti JA, Aqeel AA, Sewairi WA, Boumah CE, Kambouris M, Mayouf SA, Sheth KV, Eid WA, Dowling O, Harris J, et al: Mutation of the matrix metalloproteinase 2 gene (MMP2) causes a multicentric osteolysis and arthritis syndrome. Nat Genet 28: 261-265, 2001.

32. Evans BR, Mosig RA, Lobl M, Martignetti CR, Camacho C, Grum-Tokars V, Glucksman MJ and Martignetti JA: Mutation of membrane type-1 metalloproteinase, MT1-MMP, causes the multicentric osteolysis and arthritis disease Winchester syndrome. Am J Hum Genet 91: 572-576, 2012.

33. de Vos IJHM, Tao EY, Ong SLM, Goggi JL, Scerri T, Wilson GR, Low CGM, Wong ASW, Grussu D, Stegmann APA, et al: Functional analysis of a hypomorphic allele shows that MMP14 catalytic activity is the prime determinant of the Winchester syndrome phenotype. Hum Mol Genet 27: 2775-2788, 2018. 
34. Kröger L, Löppönen T, Ala-Kokko L, Kröger H, Jauhonen HM, Lehti $\mathrm{K}$ and Jääskeläinen $\mathrm{J}$ : A novel mutation in the matrix metallopeptidase 2 coding gene associated with intrafamilial variability of multicentric osteolysis, nodulosis, and arthropathy. Mol Genet Genomic Med 7: e802, 2019.

35. Dagher R, Saliba E,Rizkallah M, Khalife MCF and Megarbane A Multicentric osteolysis with nodulosis and arthropathy (Mona) Report of the first lebanese family. Ann Rheum Dis 77: 496-497, 2018.

36. de Vos I, Wong ASW, Welting TJM, Coull BJ and van Steensel MAM: Multicentric osteolytic syndromes represent a phenotypic spectrum defined by defective collagen remodeling. Am J Med Genet A 179: 1652-1664, 2019.

37. Vanatka R, Rouzier C, Lambert JC, Leroux C and Coussement A Winchester syndrome: The progression of radiological findings over a 23-year period. Skeletal Radiol 40: 347-351, 2011.

38. Azzollini J, Rovina D, Gervasini C, Parenti I, Fratoni A Cubellis MV, Cerri A, Pietrogrande L and Larizza L: Functional characterisation of a novel mutation affecting the catalytic domain of MMP2 in siblings with multicentric osteolysis, nodulosis and arthropathy. J Hum Genet 59: 631-637, 2014.

39. Mosig RA and Martignetti JA: Loss of MMP-2 in murine osteoblasts upregulates osteopontin and bone sialoprotein expression in a circuit regulating bone homeostasis. Dis Model Mech 6 : 397-403, 2013

40. Massague J and Obenauf AC: Metastatic colonization by circulating tumour cells. Nature 529: 298-306, 2016.

41. Svandova E, Vesela B, Lesot H, Sadoine J, Poliard A and Matalova E: FasL modulates expression of Mmp2 in osteoblasts. Front Physiol 9: 1314, 2018

42. Feng $P$, Zhang $H$, Zhang $Z$, Dai $X$, Mao $T$, Fan $Y$, Xie $X$, Wen $H$, Yu P, Hu Y and Yana R: The interaction of MMP-2/B7-H3 in human osteoporosis. Clin Immunol 162: 118-1124, 2016.

43. Pesce Viglietti AI, Arriola Benitez PC, Gentilini MV, Velasquez LN, Fossati CA, Giambartolomei GH and Delpino MV: Brucella abortus invasion of osteocytes modulates connexin 43 and integrin expression and induces osteoclastogenesis via receptor activator of NF- $\mathrm{KB}$ ligand and tumor necrosis factor Alpha Secretion. Infect Immun 84: 11-20, 2016.

44. Hong G, Zhou L, Shi X, He W, Wang H, Wei Q, Chen P, Qi L, Tickner J, Lin L and Xu J: Bajijiasu abrogates osteoclast differentiation via the suppression of RANKL signaling pathways through NF- $\kappa$ B and NFAT. Int J Mol Sci 18: 203, 2017.

45. Waning DL, Mohammad KS, Reiken S, Xie W, Andersson DC, John S, Chiechi A, Wright LE, Umanskaya A, Niewolna M, et al: Excess TGF- $\beta$ mediates muscle weakness associated with bone metastases in mice. Nat Med 21: 1262-1271, 2015.

46. Luis-Ravelo D, Antón I, Zandueta C, Valencia K, Ormazábal C, Mart ínez-Canarias S, Guruceaga E, Perurena N, Vicent S, De Las Rivas J and Lecanda F: A gene signature of bone metastatic colonization sensitizes for tumor-induced osteolysis and predicts survival in lung cancer. Oncogene 33: 5090-5099, 2014.

47. El-Farrash RA, Ali RH and Barakat NM: Post-natal bone physiology. Semin Fetal Neonatal Med 25: 101077, 2020.

48. Rhodes SD, Wu X, He Y, Chen S, Yang H, Staser KW, Wang J, Zhang $\mathrm{P}$, Jiang $\mathrm{C}$, Yokota $\mathrm{H}$, et al: Hyperactive transforming growth factor- $\beta 1$ signaling potentiates skeletal defects in a neurofibromatosis type 1 mouse model. J Bone Miner Res 28: 2476-2489, 2013.

49. Juarez $P$ and Guise TA: TGF- $\beta$ in cancer and bone: Implications for treatment of bone metastases. Bone 48: 23-29, 2011.

50. Quintero-Fabian S, Arreola R, Becerril-Villanueva E, Torres-Romero JC, Arana-Argaez V, Lara-Riegos J, Ramirez-Camacho MA and Alvarez-Sanchez ME: Role of matrix metalloproteinases in angiogenesis and cancer. Front Oncol 9: 1370, 2019.

51. Li Q, Yang J, Chen C, Lin X, Zhou M, Zhou Z and Huang Y: A novel mitochondrial targeted hybrid peptide modified HPMA copolymers for breast cancer metastasis suppression. J Control Release 325: 38-51, 2020

52. Itoh T, Tanioka M, Yoshida $\mathrm{H}$, Yoshioka $\mathrm{T}$, Nishimoto $\mathrm{H}$ and Itohara S: Reduced angiogenesis and tumor progression in gelatinase A-deficient mice. Cancer Res 58: 1048-1051, 1998.

53. Saran WR, Chierice GO, da Silva RA, de Queiroz AM, Paula-Silva FW and da Silva LA: Castor oil polymer induces bone formation with high matrix metalloproteinase-2 expression. J Biomed Mater Res A 102: 324-331, 2014.

54. Itoh $\mathrm{T}$, Ikeda $\mathrm{T}$, Gomi $\mathrm{H}, \mathrm{Nakao} \mathrm{S}$, Suzuki $\mathrm{T}$ and Itohara $\mathrm{S}$ : Unaltered secretion of beta-amyloid precursor protein in gelatinase A (matrix metalloproteinase 2)-deficient mice. J Biol Chem 272: 22389-22392, 1997.
55. Mosig RA, Dowling O, DiFeo A, Ramirez MC, Parker IC, Abe E, Diouri J, Aqeel AA, Wylie JD, Oblander SA, et al: Loss of MMP-2 disrupts skeletal and craniofacial development and results in decreased bone mineralization, joint erosion and defects in osteoblast and osteoclast growth. Hum Mol Genet 16: 1113-1123, 2007.

56. Inoue K, Mikuni-Takagaki Y, Oikawa K, Itoh T, Inada M, Noguchi T, Park JS, Onodera T, Krane SM, Noda M and Itohara S: A crucial role for matrix metalloproteinase 2 in osteocytic canalicular formation and bone metabolism. J Biol Chem 281: 33814-3324, 2006

57. Nyman JS, Lynch CC, Perrien DS, Thiolloy S, O'Quinn EC, Patil CA, Bi X, Pharr GM, Mahadevan-Jansen A and Mundy GR: Differential effects between the loss of MMP-2 and MMP-9 on structural and tissue-level properties of bone. J Bone Miner Res 26: 1252-1260, 2011.

58. Lieu S, Hansen E, Dedini R, Behonick D, Werb Z, Miclau T, Marcucio R and Colnot C: Impaired remodeling phase of fracture repair in the absence of matrix metalloproteinase-2. Dis Model Mech 4: 203-211, 2011.

59. Mumm S, Huskey M, Duan S, Wenkert D, Madson KL, Gottesman GS, Nenninger AR, Laxer RM, McAlister WH and Whyte MP: Multicentric carpotarsal osteolysis syndrome is caused by only a few domain-specific mutations in MAFB, a negative regulator of RANKL-induced osteoclastogenesis. Am J Med Genet A 164A: 2287-2293, 2014.

60. Lazarus S, Tseng HW, Lawrence F, Woodruff MA, Duncan EL and Pettit AR: Characterization of normal murine carpal bone development prompts Re-evaluation of pathologic osteolysis as the cause of human Carpal-tarsal osteolysis disorders. Am J Pathol 187: 1923-1934, 2017.

61. Russo MV, Latour LL and McGavern DB: Distinct myeloid cell subsets promote meningeal remodeling and vascular repair after mild traumatic brain injury. Nat Immunol 19: 442-452, 2018

62. Rundhaug JE: Matrix metalloproteinases and angiogenesis. J Cell Mol Med 9: 267-285, 2005.

63. Cheng XW, Kuzuya M, Nakamura K, Maeda K, Tsuzuki M, Kim W, Sasaki T, Liu Z, Inoue N, Kondo T, et al: Mechanisms underlying the impairment of ischemia-induced neovascularization in matrix metalloproteinase 2-deficient mice. Circ Res 100: 904-913, 2007.

64. Trivedi A, Zhang H, Ekeledo A, Lee S, Werb Z, Plant GW and Noble-Haeusslein LJ: Deficiency in matrix metalloproteinase-2 results in long-term vascular instability and regression in the injured mouse spinal cord. Exp Neurol 284: 50-62, 2016.

65. Peng Y, Wu S, Li Y and Crane JL: Type H blood vessels in bone modeling and remodeling. Theranostics 10: 426-436, 2020.

66. Yang P, Lv S, Wang Y, Peng Y, Ye Z, Xia Z, Ding G, Cao X and Crane JL: Preservation of type $\mathrm{H}$ vessels and osteoblasts by enhanced preosteoclast platelet-derived growth factor type BB attenuates glucocorticoid-induced osteoporosis in growing mice. Bone 114: 1-13, 2018.

67. Romeo SG, Alawi KM, Rodrigues J, Singh A, Kusumbe AP and Ramasamy SK: Endothelial proteolytic activity and interaction with non-resorbing osteoclasts mediate bone elongation. Nat Cell Biol 21: 430-441, 2019.

68. Elefteriou F: Impact of the autonomic nervous system on the skeleton. Physiol Rev 98: 1083-1112, 2018.

69. Reid IR, Baldock PA and Cornish J: Effects of leptin on the skeleton. Endocr Rev 39: 938-959, 2018.

70. Mazor R, Friedmann-Morvinski D, Alsaigh T, Kleifeld O, Kistler EB, Rousso-Noori L, Huang C, Li JB, Verma IM and Schmid-Schonbein GW: Cleavage of the leptin receptor by matrix metalloproteinase-2 promotes leptin resistance and obesity in mice. Sci Transl Med 10: eaah6324, 2018.

71. Lee WC, Guntur AR, Long F and Rosen CJ: Energy metabolism of the osteoblast: Implications for osteoporosis. Endocr Rev 38: 255-266, 2017.

72. Fernandez-Patron C, Kassiri Z and Leung D: Modulation of systemic metabolism by MMP-2: From MMP-2 deficiency in Mice to MMP-2 deficiency in patients. Compr Physiol 6: 1935-1949, 2016.

73. Fernandez-Patron C, Stewart KG, Zhang Y, Koivunen E, Radomski MW and Davidge ST: Vascular matrix metalloproteinase-2-dependent cleavage of calcitonin gene-related peptide promotes vasoconstriction. Circ Res 87: 670-676, 2000

74. Kwan JA, Schulze CJ, Wang W, Leon H, Sariahmetoglu M, Sung M, Sawicka J, Sims DE, Sawicki G and Schulz R: Matrix metalloproteinase-2 (MMP-2) is present in the nucleus of cardiac myocytes and is capable of cleaving poly (ADP-ribose) polymerase (PARP) in vitro. FASEB J 18: 690-692, 2004

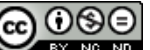

This work is licensed under a Creative Commons Attribution-NonCommercial-NoDerivatives 4.0 International (CC BY-NC-ND 4.0) License. 